\title{
Design and Implementation of a Multihoming-Based Scheme to Support Mobility Management in NEMO
}

\author{
Shayla Islam ${ }^{1}$ - Aisha-Hassan Abdalla Hashim ${ }^{1}$. \\ Mohamed Hadi Habaebi ${ }^{1}$. Mohammad Kamrul Hasan ${ }^{1}$
}

Published online: 23 November 2016

(C) Springer Science+Business Media New York 2016

\begin{abstract}
With the worldwide deployment of different wireless access technologies and expanding requests for worldwide Internet accessibility, Network Mobility (NEMO) is turning out to be all the more requesting innovation. Therefore, NEMO Basic Support Protocol (NEMO BSP) has been anticipated by the NEMO Working Group for the continual worldwide connectivity of each node within the mobile network. Yet, NEMO BSP experiences from higher handoff delay and packet loss. Thus, fails to provide seamless handoff. This issue can be resolved by introducing multihoming concept in NEMO. The reason is to support concurrent use of multiple interfaces on a single Mobile Router. Therefore, the main objective of this paper is to propose an innovative Multihoming-based scheme to support Mobility management in Proxy NEMO (MM-PNEMO) environment. In addition, the performance is assessed utilizing simulation approach to certifying the applicability as well as the efficacy of the suggested scheme. The performance metrics used for evaluation are namely handoff delay, packet loss, packet delivery ratio, as well as throughput. The simulation is done using NS-3 network simulator. The simulation outcomes demonstrate that the proposed MM-PNEMO scheme outperforms the standard NEMO BSP and PNEMO in terms of packet loss (less than 1\%) and handoff delay (average improvement by $76 \%$ ).
\end{abstract}

Keywords NEMO $\cdot$ NEMO BSP · PNEMO $\cdot$ Mobility management $\cdot$ Multihoming

Shayla Islam

iium19612@hotmail.com

1 Department of Electrical and Computer Engineering, International Islamic University Malaysia, Jalan Gombak 53100, Selangor, Malaysia 


\section{Introduction}

As different wireless access technologies are being available around the world, the coming generation of access networks is purposefully focused towards IP-based networks. IPbased networks will positively empower the whole of mobility as well as Internet availability to mobile strategies. Furthermore, mobile networks are capable of combining with IP-empowered devices such as laptops, transports and so on [1, 2]. Therefore, Network Mobility Basic Support Protocol (NEMO-BSP) has been institutionalized via Internet Engineering Task Force (IETF) [3]. This is to enhance continuous Internet availability to Mobile Network Nodes (MNNs) in such networks. Simplicity has been identified as the most imperative feature of this protocol [4-7]. But in accordance with NEMO BSP, the Mobile Router (MR) utilizes single Interface to attach to the access link. The communication of the moving network pass through the bidirectional tunnel among MR as well as its Home Agent (HA). MR requires changing its point of attachment to the network when it moves among different access network. Thus, utilizing a solitary Care of Address (CoA) makes the issue of system failure prompting a shutdown of Internet connection. This happens just if that particular single interface connection stops and no alternatives stay to keep the availability. Therefore, the execution of handoff process typically takes lengthier period. This may result in performance deterioration of the applications running on Mobile Network Nodes (MNNs). Therefore, the IETF group (i.e. Mobility Extensions (MEXT)) introduces multihoming concept as an extension of NEMO BSP in which it proposed Multiple CoA (MCoA) schemes with IP Flow Bindings [8, 9]. This is to support simultaneous use of multiple interfaces on a single MR (Multihomed MR) to avoid a single point of failure during handoff. However, this scheme inherits the standard MIPv6 drawbacks such as creating a bi-directional tunnel as well as exchanging the mobility-related L3 signaling messages through the wireless access link [10]. This can be addressed by integrating the flow bindings with PMIPv6 [18-21]. However, adding this function of IP flow bindings during inter-technology handoff on PMIPv6 domain in NEMO (PNEMO) network is still a challenging issue as consideration has to be given on both MR and its MNNs in the PNEMO environment $[5,6,11,12]$. MRs are not capable of using multiple interfaces simultaneously during the movement among different access technologies. Thus, applications flows are not capable of using the access network which is mostly appropriate to its characteristics. This eventually degrades the network performance (i.e. lower throughput and packet delivery ratio) as well as increase handoff delay and packet loss. Therefore, a multihoming-based scheme is required in PNEMO environment to address these issues as well as to enable the flow bindings in PNEMO.

The main contribution of this paper is given below:

(a) An innovative Multihoming-based scheme is proposed to support Mobility management in PNEMO (MM-PNEMO).

- Enhanced flow-based routing in PNEMO environment to support fast registration mechanism. As a result, MRs are capable of improving network performance by using multiple interfaces simultaneously during the movement among different access technologies.

(b) The performance of the proposed MM-PNEMO scheme is assessed via simulation approach. In simulation part, implement an extensive simulation scenario as well as the modeling of the proposed MM-PNEMO scheme on PMIPv6 domain using NS-3 simulator version 3.12.1. 
- Simulation outcomes show that the proposed scheme has attained notable improvement (maximized the network throughput and packet delivery ratio as well as decrease handoff delay and packet loss) compared to the standard NEMO BSP and PNEMO scheme.

The remaining part of this paper is structured as follows: Sect. 2 provides related work which is followed by the detailed operation of the proposed scheme in Sect. 3. Then, the performance of the MM-PNEMO scheme is appraised through simulation in Sect. 4. Afterward, the analysis and discussion of the results achieved from the simulation are provided in Sect. 5. Finally, the paper is concluded in Sect. 6.

\section{Related Work}

NEMO BSP involves several extensions which support persistent Internet connectivity across different access networks. For the purpose of this paper, the discussion will emphasize on multihoming-based proposals to support mobility in PNEMO environment.

In mobile environment perspective, there have been some proposals linked to networkbased localized mobility protocol manipulating the fast handoff feature in Proxy NEMO (PNEMO) environment $[5,6,13,14]$. The novelty of these paper is that, MR is equipped for upgrading the information of its current state amid handoff without making its individual mobility related signaling. Also, the lightweight mechanisms offers the enhanced performance by maintaining a strategic distance from the DAD procedure for every handoff. Since the advanced handoff preparation strategy is applied in Fast PNEMO (FPNEMO) scheme, thus it decreases packet loss as well as delay during handoff within intra mobility atmosphere. However, in the case of PNEMO network, as the total number of MNNs rises, the tunneling burden increases. The tunnel is incredibly overloaded once several MNNs send or accept excessive traffic density that effects in severe congestion and disruption of session continuity. Moreover, integrating multihoming technique with Fast PNEMO (F-PNEMO) during inter-mobility environment is yet an open research issue.

In addition to that, the authors [7, 15] have proposed multiple mobile routers based handoff scheme to comprise several HAs in where the approach extends several Mobile Routers (MRs). Each HA belongs to various organizational domains which offer the smooth handoff for the vehicles in heterogeneous networks. It can be observed from the simulation results that, these strategies are capable of lessening packet loss without service disturbance in the course of handoff. Conversely, signaling overhead is the major shortcomings of this scheme. This leads to increases the handoff cost for its extra signaling messages and tunneling overhead due to using multiple MRs.

Moreover, a Multihoming-based Vertical handoff scheme on PMIPv6 (MV-PMIPv6) has been suggested for node movement [16]. The authors consider that, every interface of a Mobile Node (MN) has its own protocol stack with physical, data link, and network layer. Therefore, at the time of movement from one access network to another, it is possible to diminish handoff delay with packet loss by introducing a newly defined PBU and PBA messages. Supporting 'make-before-break' handoff in Layer 3 (L3) is the positive side of this suggested scheme. Yet, MV-PMIPv6 mechanism ought to consider in NEMO environment where MR handle the movability for the benefit of all MNNs.

A seamless multi-interfaced based handoff scheme have studied by proposing a path selection algorithm to discover the optimal path amongst multiple interfaces [17]. Through 
investigation, it is shown that multi-interfaced MR is fit for increasing the MR handoff performance over the different access network. Nevertheless, it is important to focus on the implementation cost due to employing multiple interfaces.

The authors in [18-21] have projected few proposals on PMIPv6 domain that support flow enabled routing. However, the application of these proposals in PNEMO environment stills a dynamic research area. Existing multihoming-Based scheme are summarized in Table 1.

\section{Proposed MM-PNEMO Scheme}

With the aim of taking the benefit of using multiple interfaces, this section provides the detailed operation of the proposed Multihoming-based scheme in order to support Mobility Management in PNEMO (MM-PNEMO) environment. According to the MM-PNEMO scheme, the Flow-based Local Mobility Anchor Point (FLMA) and the Serving MR (SMR) act like an Local Mobility Anchor Point (LMA) and Mobile Router (MR) in PNEMO environment. In the proposed MM-PNEMO scheme, the Current Flow-enabled MR (CFMR) and the New Flow-enabled MR (NFMR) are used as replacement of Mobile

Table 1 Summary of existing multihoming-based mobility management proposals

\begin{tabular}{|c|c|c|}
\hline Existing research work & Strength & Weakness \\
\hline $\begin{array}{l}\text { Lightweight NEMO Within } \\
\text { PMIPv6 for Transportation } \\
\text { Systems (PNEMO) }[5,6]\end{array}$ & $\begin{array}{l}\text { Does not essential an MR to } \\
\text { generate its own mobility } \\
\text { signaling to update the current } \\
\text { location }\end{array}$ & $\begin{array}{l}\text { As the total number of MNNs rises, } \\
\text { the tunneling burden increases }\end{array}$ \\
\hline $\begin{array}{l}\text { Enhanced Fast Handoff for } \\
\text { NEMO within PMIPv6 for } \\
\text { intelligent transportation } \\
\text { systems [13] }\end{array}$ & $\begin{array}{l}\text { Reduce tunneling burden through } \\
\text { applying early registration }\end{array}$ & $\begin{array}{l}\text { Should integrate flow based routing } \\
\text { within NEMO on PMIPv6 to } \\
\text { provide seamless handoff as well } \\
\text { as to avoid buffering cost }\end{array}$ \\
\hline $\begin{array}{l}\text { Efficient NEMO support } \\
\text { scheme for proxy MIPv6 [14] }\end{array}$ & $\begin{array}{l}\text { Reduce location update cost and } \\
\text { packet tunneling cost }\end{array}$ & $\begin{array}{l}\text { Need to include cost evaluation } \\
\text { criteria for each NEMO entities }\end{array}$ \\
\hline $\begin{array}{l}\text { Multiple mobile routers based } \\
\text { seamless handover } \\
\text { scheme for multihomed } \\
\text { NEMO }[7,15]\end{array}$ & $\begin{array}{l}\text { Reduce packet loss with no service } \\
\text { disruption. Moreover, enhance } \\
\text { the band width, network coverage } \\
\text { as well as reliability of NEMO }\end{array}$ & $\begin{array}{l}\text { Contains some overhead that } \\
\text { includes the cost due to maintain } \\
\text { additional signaling messages for } \\
\text { multiple MRs }\end{array}$ \\
\hline $\begin{array}{l}\text { Multihoming-based Vertical } \\
\text { handoff scheme on PMIPv6 } \\
\text { (MV-PMIPv6) [16] }\end{array}$ & $\begin{array}{l}\text { Reduce vertical handoff delay and } \\
\text { packet loss }\end{array}$ & $\begin{array}{l}\text { Recommended to support flow- } \\
\text { enabled mobility to provide } \\
\text { Quality of Service (QoS) } \\
\text { successfully }\end{array}$ \\
\hline $\begin{array}{l}\text { Experimentation and } \\
\text { performance analysis of } \\
\text { multi-interfaced mobile } \\
\text { router scheme [17] }\end{array}$ & $\begin{array}{l}\text { Reduce service disruption time } \\
\text { with low packet loss }\end{array}$ & $\begin{array}{l}\text { Should focus on deployment as } \\
\text { well as signaling overhead since } \\
\text { to use multiple interfaces along } \\
\text { with security issues }\end{array}$ \\
\hline $\begin{array}{l}\text { Network-based flow binding } \\
\text { mechanism }[9,10,18-21]\end{array}$ & $\begin{array}{l}\text { Provide a wide range of Quality of } \\
\text { Service (QoS) }\end{array}$ & $\begin{array}{l}\text { Need to focus on Inter -technology } \\
\text { handoff in NEMO }\end{array}$ \\
\hline $\begin{array}{l}\text { Cost analysis of seamless IP } \\
\text { diversity based NEMO } \\
\text { entities }[1,2,4]\end{array}$ & $\begin{array}{l}\text { Capable to reduce the signaling } \\
\text { cost for update the location }\end{array}$ & $\begin{array}{l}\text { Recommends to use flow bindings } \\
\text { in NEMO to get the multihoming } \\
\text { features completely }\end{array}$ \\
\hline
\end{tabular}


Access Gateway (MAG1) and Mobile Access Gateway (MAG2). The CFMR and NFMR are liable for identifying the SMR's movements among Different Access Technology Type (DATT). They are also responsible to differentiate and attain the Mobile Network Prefix (MNP) and Home Network Prefix (HNP) from an acknowledgment message which is directed from the local HA (FLMA). Besides, CFMR and NFMR exchanges the context of the SMR via Layer 2 (L2) triggering mechanism. A reference framework for the network entities is depicted in Fig. 1. The proposed architecture is enhanced to support the networkbased localized mobility protocol in NEMO environment.

In the proposed MM-PNEMO scheme, the CFMR as well as NFMR exchanges Handover Initiations (HI) and Handover Acknowledgement (HAck) messages earlier to the L2 handoff. This is to efficiently support SMR-initiated flow-based routing in PNEMO environment. The HI message contains the accumulated information of the SMR such as the SMR ID, Home Network Prefix (HNP), Mobile Network Prefix (MNP), FLMA address, Flow ID, priority, type of application flow, and so on. This accumulated information helps to enable the NFMR to redirect a binding registration message with MNP option of SMR to FLMA for accomplishing a location update process. In order to perform fast registration, an Early Proxy Binding Update (EPBU) and Early Proxy Binding Acknowledgement messages (EPBA) are encapsulated inside HI and HAck messages respectively. Moreover, a new mobility option, termed as Flow-based Mobile Network Prefix (FMNP) option is included in the EPBU and EPBA messages. This is to notify the interfaces present status and to request for performing the flow-based routing during inter-technology handoff in PNEMO environment. The details of these messages will be explained later in this section. A comprehensive flow diagram of the proposed MM-PNEMO scheme is represented in Fig. 2.

The proposed scheme offers the location update procedure in order to distinct the new connection of the SMR that is termed as 'fast registration' process from the particular flow movement which is entitled as 'flow-based routing' process (as shown in Fig. 2). As soon as the NFMR identifies the new attachment of SMR on the target network, the NFMR

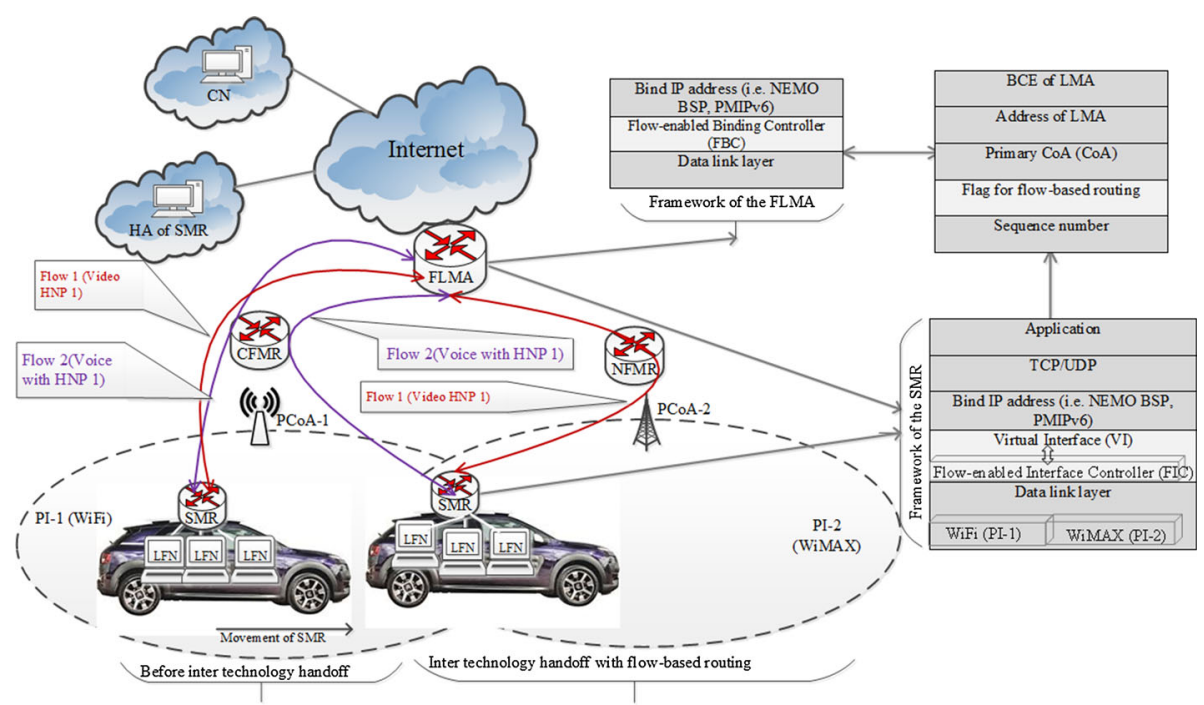

Fig. 1 Reference framework of MM-PNEMO scheme 


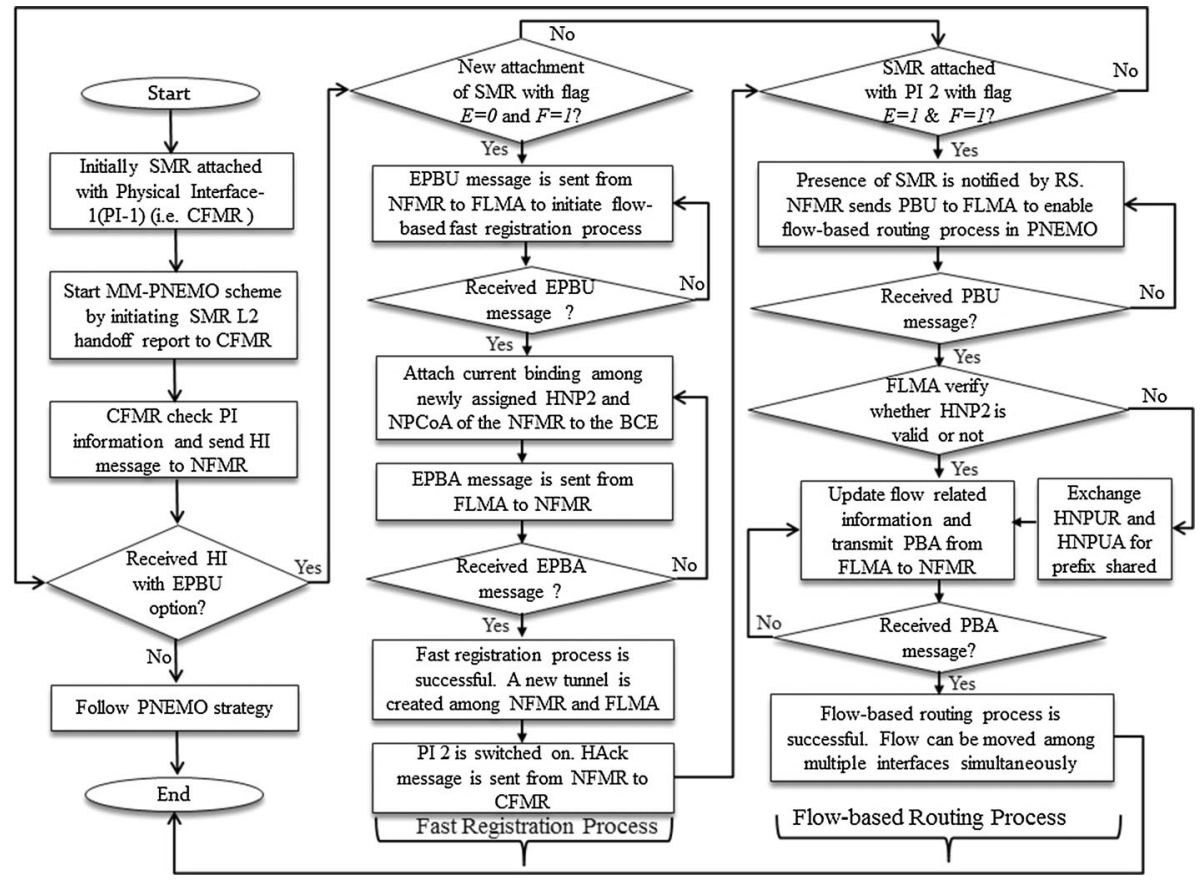

Fig. 2 Flow diagram of the proposed MM-PNEMO scheme

sends EPBU message via HI message to the FLMA for initiating the fast registration process without enabling flow-based routing information. During fast registration mechanism, the FLMA accomplishes few tasks for the new attachment such as allocating a new Home Network Prefix (HNP) and Mobile Network Prefix (MNP) of the Physical Interface 2 (PI 2), building new Binding Catch Entry (BCE) for the SMR as well as creating a new tunnel among the FNMR and the FLMA. If the NFMR identifies that the SMR is connected to the new PI (PI 2), it transmits PBU message containing FMNP option to FLMA in order to initiate flow-based routing process. Once the process is completed successfully, a PBA message is directed from the FLMA to the NFMR. However, if the network entity (CFMR, NFMR or FLMA) is not informed about the HI message with encapsulated EPBU option, the SMR will initiate PNEMO handoff process.

\subsection{Extended Message Format}

The Early Proxy Binding Update (EPBU) message is an extension of Proxy Binding Update (PBU) message. Some flags are included in EPBU message to support the proposed MM-PNEMO scheme as shown in Fig. 3. According to MM-PNEMO, $R$ flag is used for specifying Mobile network Node (MNN) mobility or an SMR mobility. If $R$ bit is set to 1 , the FLMA assumes that it forward the data packets bounded for the NEMO to the SMR (not to the MNN). Besides, EPBU message added another flag $(E=0)$ to inform the Flowbased Local Mobility Anchor Point (FLMA) that the EPBU is a fast registration message. When $E$ is fixed to 1 , then the scheme follows the usual PBU process in the proposed scheme. Moreover, FMNP option (as depicts in Fig. 3) is included in the EPBU message 


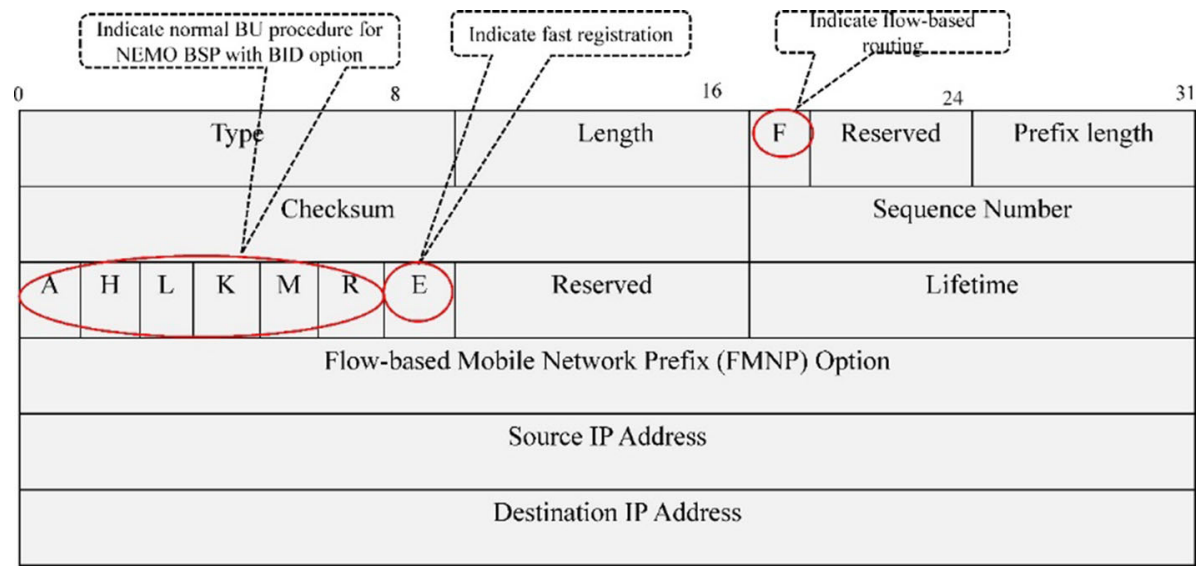

Fig. 3 Extended EPBU message

with setting flag $F=1$ in order to initiate the flow-based routing in the MM-PNEMO scheme. It contains the HNPs and the MNPs that are listed in the new link of the SMR.

The Home Network Prefix Update Request (HNPUR) and Home Network Prefix Update Acknowledgement (HNPUA) messages are similar to Flow Mobility Initiate (FMI) and Flow Mobility Acknowledgement (FMA) messages [11]. Usually, these two messages are considered as a Mobility Header (MH) message. In order to notify about the changes of few parameters concerning flow-based routing, HNPUR message can be transmitted by the FLMA to access gateways (NFMR or CFMR). It is possible to use HNPUR message as a new message. Though, in the proposed scheme, the existing EPBA message is applied as an HNPUR message with setting some flags $(E=1, F=1)$ by the FLMA as depicted in Fig. 4. This is to indicate that HNPUR is a retransmitted message and FLMA is waiting for acknowledging message from NFMR. The same way as HNPUA, the existing EPBU message is applied as an HNPUA message with setting $T$ flag $(T=1)$ by the FLMA. This is to indicate that HNPUR is an acknowledgment message.

The proposed mobility option named as Flow-based MNP (FMNP) option is included in the EPBU and EPBA messages to describe the prefix related information between the FLMA and NFMR. This option holds flow related information that allows the Flow-based Local Mobility Anchor Point (FLMA) to provide the Home Network Prefix (HNPs) and Mobile Network Prefix (MNPs) of the Serving Mobile Routers (SMRs) additional links. The HNP and MNP enclosed with this FMNP option have to be directed to the network entities only, not for the mobility entities (SMR). In the proposed scheme, the New Flowenabled MR (NFMR) attaches and transmits this option in an EPBU message to enable

\begin{tabular}{|c|c|c|c|c|c|c|c|}
\hline 0 & & & & & & & \\
\hline Type & Length & Reserved & $\mathrm{K}$ & $\mathrm{R}$ & E & $\mathrm{F}$ & Status \\
\hline \multicolumn{2}{|c|}{ Lifetime } & \multicolumn{6}{|c|}{ Sequence Number } \\
\hline
\end{tabular}

Flow-based Mobile Network Prefix (FMNP) Option

Fig. 4 Extended EPBA message 
flow-based routing. The FLMA also attaches this option with the EPBA message for notifying the NFMR about the importance of the request. The extended Flow-based MNP (FMNP) option is shown in Fig. 3. The $F$ flag is involved in the FMNP option to show the FLMA that the SMR is able to maintain flow enabled routing. When $F=1$, the SMR will perform flow-based routing. However, when $F=0$, the SMR is not able to support flowbased routing.

\section{Simulation Evaluation in NS 3}

This section provides simulation scenario as well as the modeling of the proposed MMPNEMO scheme on PMIPv6 domain using NS-3 simulator version 3.12.1 [22]. The motivation behind the simulation was to check the effect that the proposed architecture would essence to both the system as well as the application flows. For simplicity, it is anticipated that the FLMA in MM-PNEMO is placed in the similar place as LMA in PNEMO and HA in NEMO BSP. Likewise, it is presumed that CFMR and NFMR in MMPNEMO are located at the identical place as MAG1 and MAG 2 in PNEMO and AR in NEMO BSP as depicted in Fig. 5. Besides, it is also assumed that SMR is connecting with

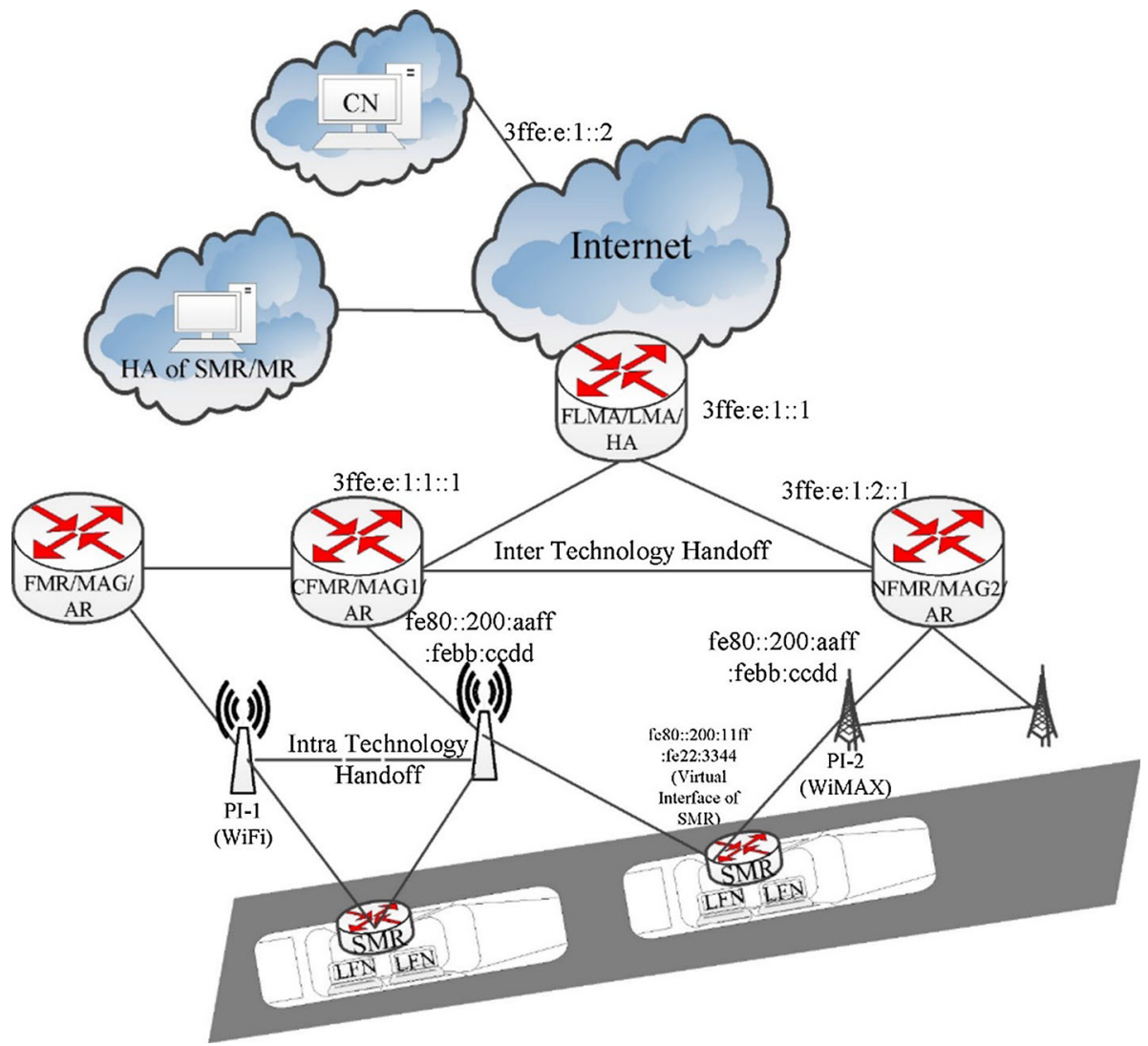

Fig. 5 Simulation scenario in NS 3 simulator 
one Corresponding Node (CN) via the local HA (i.e. FLMA). The performance assessment is done mostly based on substantial metrics are mainly handoff delay, packet loss, packet delivery ratio, and throughput, respectively. The following subsection is going to present the simulation environment in details.

The performance of the proposed scheme is then benchmarked with that of the NEMO BSP and PNEMO scheme [3, 5]. PNEMO is compared with MM-PNEMO since both schemes integrates firmly-entrenched PMIPv6 which is a network-based localized mobility protocol in NEMO to address mobility issues [11]. In contrast, NEMO BSP is the standard protocol for any mobility support management aiming at NEMO network and it is also an improved version of the Mobile IPv6.

\subsection{Simulation Environment}

This section gives three non-indistinguishable scenarios that are defined to assess the performance of the proposed flow-based fast registration mechanism are mainly: scenario 1, scenario 2 and scenario 3. The details of each scenario are specified in Table 2.

The network animation of the MM-PNEMO scheme is illustrated in Fig. 6 in which the SMR is equipped with two heterogeneous wireless access technologies are mainly WiFi and WiMAX network environment during inter-technology handoff. The configuration utilized for both access technologies were defined in NS-3.12.1. The speed for a wired connection is assumed to be $50 \mathrm{Mbps}$ whereas the link delay is assumed to be $0.1 \mathrm{~ms}$. The maximum link speed for $\mathrm{WiFi}$ and WiMAX link are $54 \mathrm{Mbps}$ as well as $75 \mathrm{Mbps}$, respectively. Firstly, the SMR is linked to the PI1 (WiFi access network) whereas the PI2 (WiMAX access network) is idled. There are two UDP application flows, including flow 1 as well as flow 2 as shown in Fig. 1. The commence times of both application flows are different in light of the scenario to confirm the proposed flow enabled multihoming-based handoff cases. The aggregate simulation time is $15 \mathrm{~s}$ with 30 SMRs that were moving among different access networks. The constant velocity mobility model was utilized to validate the performance of the MM-PNEMO architecture via changing the number of SMRs, as well as SMRs speed. In this simulation, the speed is varied from 10 to $60 \mathrm{~m} / \mathrm{s}$ with a specific end goal to check if there is any performance influences on the proposed architecture with different speeds. Moreover, NetAnim and gnuplot are utilized to investigate, visualize or process the information gained through simulation.

Table 2 Considered simulation scenario

\begin{tabular}{ll}
\hline Phase & Event \\
\hline $\begin{array}{l}\text { Scenario 1 } \\
\text { (NEMO BSP) }\end{array}$ & Activate PI1 while deactivate PI2 \\
$\begin{array}{ll}\text { Scenario 2 } \\
\text { (PNEMO) }\end{array}$ & $\begin{array}{l}\text { Both access network (WiMAX as well as WiFi) are triggered } \\
\text { Scenario 3 }\end{array}$ \\
$\begin{array}{l}\text { SM-PNEMO can use only single interfaces to which it is presently attached } \\
\text { scheme) }\end{array}$ & $\begin{array}{l}\text { Both access network (WiMAX as well as WiFi) are triggered } \\
\text { SMR requests to move flow 1 to WiMAX }\end{array}$ \\
& SMR is capable of employing both access networks concurrently during inter- \\
& system handoff
\end{tabular}




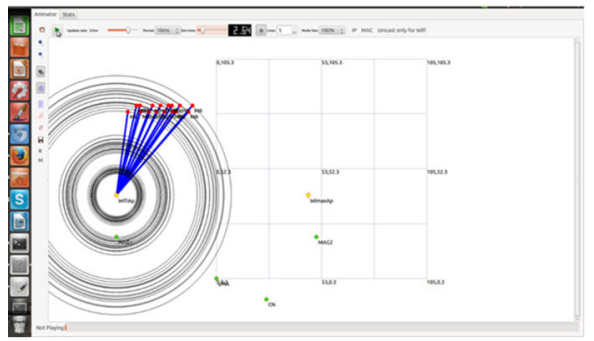

(a)

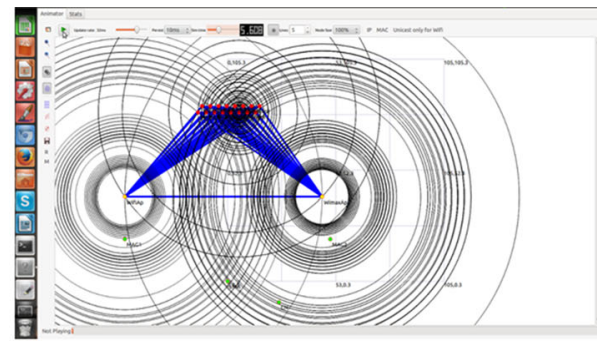

(b)

Fig. 6 Network animation in the proposed scenario. a SMR initially attached with WiFi. b SMR attached with WiFi and WiMAX

\subsection{Simulation Parameters}

The parameters which have been used to perform the simulation of proposed scheme are given in Table $3[19,22]$ :

\section{Result and Discussion}

This section covers the simulation outcome which illustrates the performance of the proposed MM-PNEMO scheme (scenario 3 in Table 2) in comparison with NEMO BSP and PNEMO scheme (scenario 1 and scenario 2 in Table 2).

Figures 7, 8 and 9 represent the average handoff delay as a function of time, the number of SMR and speed for the three scenarios. From these plotted figures, it is indicated the average handoff delay for scenario 1, scenario 2 and the MM-PNEMO scheme at the time

Table 3 System parameters that used for simulation

\begin{tabular}{ll}
\hline Parameter & Value \\
\hline Simulator & NS3.12.1 \\
Operating system & Linux Ubuntu 12.04 \\
Simulation time & {$[0-15] \mathrm{s}$} \\
Packet size & 1024 bytes \\
Data packet rate & $5 \mathrm{Mbps}$ \\
No. of SMR & $10-30$ \\
SMR speed & $10-60 \mathrm{~m} / \mathrm{s}$ \\
No. of connections per SMR & 2 \\
Total number of scenarios & 3 \\
Types of technologies supported by each SMRs & WiFi and WiMAX \\
Speed of WiFi link & $54 \mathrm{Mbps}$ \\
Speed of WiMAX link & $75 \mathrm{Mbps}$ \\
Pause time & $12 \mathrm{~ms}$ \\
Mobility model & Constant velocity mobility model \\
\hline
\end{tabular}




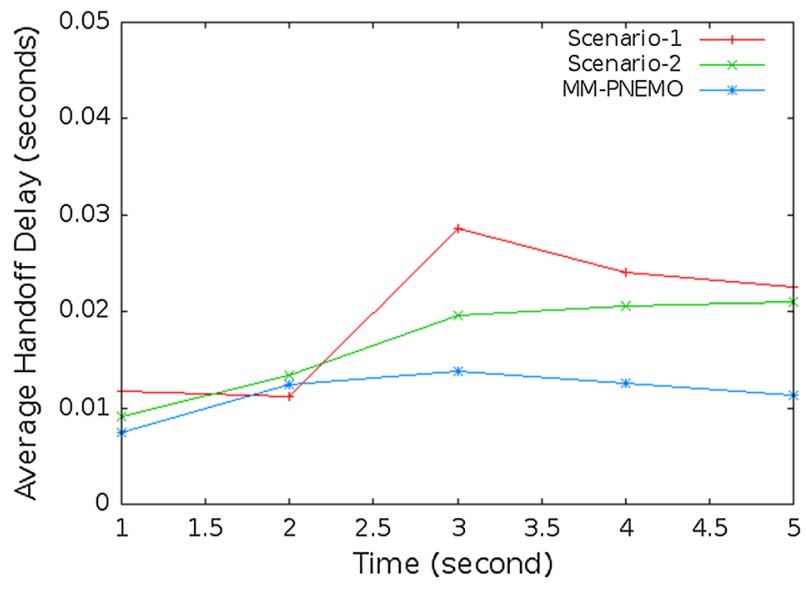

Fig. 7 Average handoff delay versus time

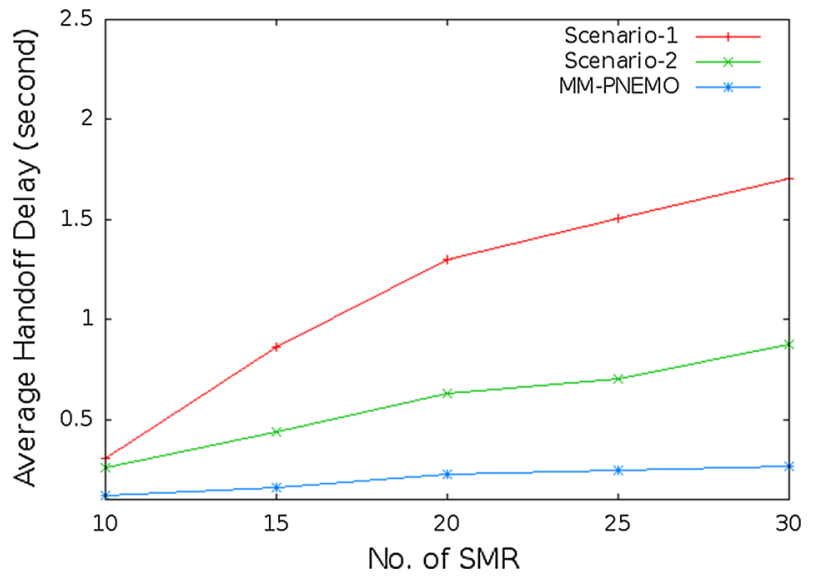

Fig. 8 Average handoff delay versus number of SMR

of SMR movements from the coverage of WiFi to WiMAX network. Analyzing Fig. 7, it can be noticed that there is a gain in the average handoff delay in each scenario with changing the time from 2 to $3 \mathrm{~s}$ during intra-movement. This outcome is linked with the total number of handoff happened as well as the network prominence at the period of the handoff during movement among different access networks. Comparing these results, it is also observed that, single-interfaced SMR in scenario 1 (speed $=30 \mathrm{~m} / \mathrm{s}$ ) and scenario 2 (speed $=40 \mathrm{~m} / \mathrm{s}$ ) experiences an additional delay. This is because they need to wait for the access router to notify the CFMR to send the packet. Likewise, the SMR requires informing HA for each movement which leads to a higher handoff delay in scenario 1. In contrast, as the FLMA handles all the local mobility, hence the number of handoffs is not high in the proposed MM-PNEMO and PNEMO scheme. This results in lower handoff delay. Consequently, MM-PNEMO also outperforms PNEMO by taking benefit of fast registration mechanism. The handoff delay in the MM-PNEMO scheme (speed $=60 \mathrm{~m} / \mathrm{s}$ ) is less (.011 s.) compared to NEMO BSP (.022 s.) and PNEMO (.021 s.). 


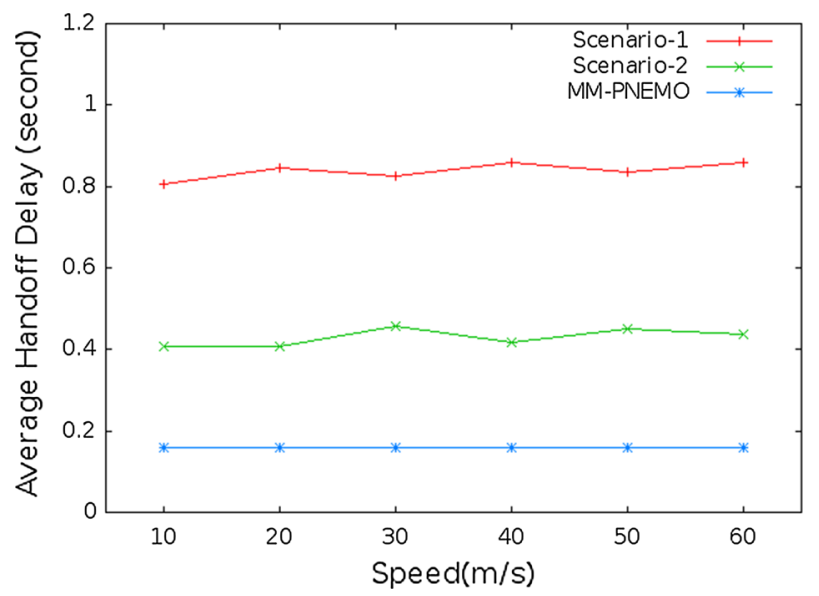

Fig. 9 Average handoff delay versus speed

Similarly, the variation of average handoff delay during inter-technology handoff for all scenarios is illustrated in Figs. 8 and 9 respectively as a function of the number of SMR and speed. It is apparent from both figures that, handoff delay of each scheme deteriorates linearly with growing the number of SMR and speed. Yet, the delay of the MM-PNEMO scheme shows smaller (.20 s) compared to scenario 1 (1.2 s) and scenario $2(.51 \mathrm{~s})$. This is due to the simultaneous movement of data flows via PI-1 and PI-2 during inter-system handoff. This lead to network performance improvement.

The effect of the changing the time on packet loss during handoff is illustrated in Figs. 10 and 11 respectively for each scenario. Figure 10 shows that, the SMR handoff does not performed at time $=0 \mathrm{~s}$. It is also apparent from Fig. 10, the time is varied from 1 to $5 \mathrm{~s}$. Looking at the outcome of Fig. 10, it is noted that there is a large variation in packet loss among MM-PNEMO (speed $=60 \mathrm{~m} / \mathrm{s}$ ) with scenario 1 (speed $30 \mathrm{~m} / \mathrm{s}$ ) and scenario $2(40 \mathrm{~m} / \mathrm{s})$. The maximum number of packet loss for MM-PNEMO, scenario 1

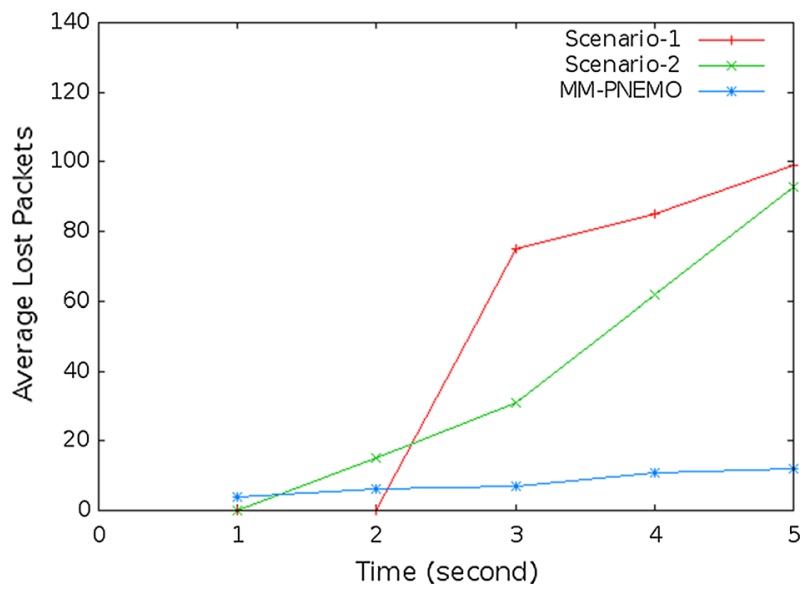

Fig. 10 Packet loss versus time 


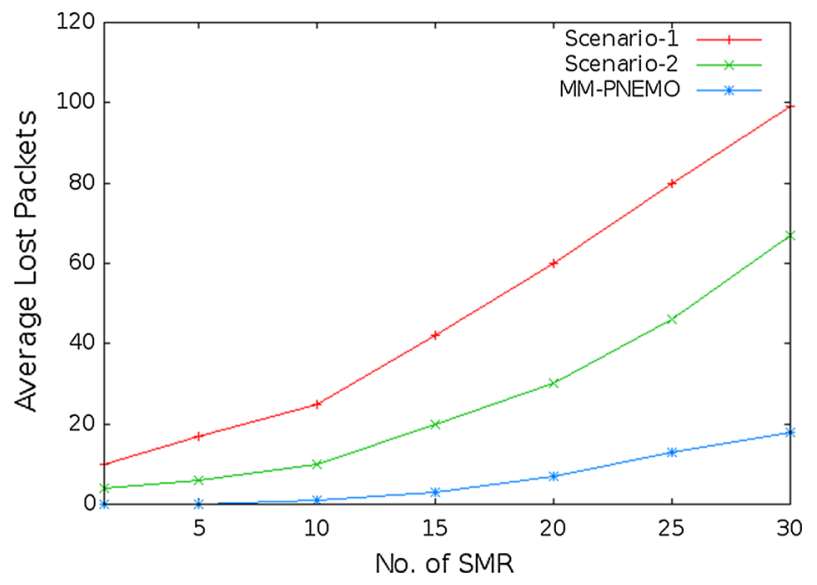

Fig. 11 Packet loss versus number of SMR

and scenario 2 are 6, 30 and 60 respectively. The outcomes also illustrate that the proposed MM-PNEMO is not much influenced by the increased time and speed. The impact of the increased number of SMR on the packet loss during handoff are illustrated in Fig. 11 where the number of SMR is varied from 1 to 30 and speed is kept constant (speeds at $30 \mathrm{~m} / \mathrm{s}$ ) for each scenario. It is also obvious that the proposed scheme experiences a lower packet loss compared to that of scenario 1 and scenario 2. This is because applying multihoming features in PNEMO environment has a supportive influence on the packet loss for the MM-PNEMO scheme.

Similarly, the packet delivery ratio in percentage is described in Fig. 12 for all the scenarios. Here, the number of SMR is set as 30 for a different speed. It is observed from the results that there is a gain in the packet delivery ratio for all scenarios with changing the time from 1 to $2 \mathrm{~s}$. However, the packet delivery ratio for scenario 1 and scenario 2 decreases as the time increases from 6 to $10 \mathrm{~s}$. It can be seen that, the MM-PNEMO

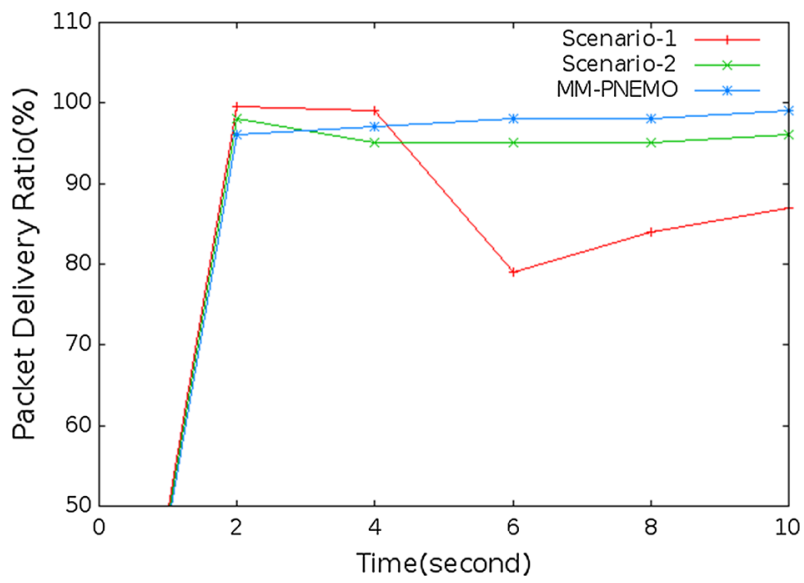

Fig. 12 Packet delivery ratio versus time 
scheme produces maximum packet delivery ratio compared to scenario 1 and scenario 2 . Moreover, it is also observed that the first scenario produces slightly more packet loss compared to the second scenario. It is probable that the packet loss increases for using single access technology in NEMO BSP and PNEMO during handoff.

Taking into consideration of 30 SMRs with speed $=30 \mathrm{~m} / \mathrm{s}$, the proposed MMPNEMO scheme provide maximum throughput of around $631 \mathrm{kbps}$ with changing time from 0 to $10 \mathrm{~s}$. In addition, the lower rate of lost packet of the proposed scheme does not relevantly affect the throughput of the system, as appeared in Fig. 13 as well as Table 4. As greater throughput leads better Internet availability, thus, it is obviously seen from Fig. 13 that, the MM-PNEMO scheme takes out unrivaled performance compared to scenario 1 as well as scenario 3. Consequently, it is summed up that, utilizing multihoming procedure to bolster mobility management in PNEMO can devour network resources competently.

\subsection{Discussion}

In this paper, an innovative Multihoming-based Mobility management scheme in PNEMO environment (MM-PNEMO) has proposed to support continuous Internet connectivity during inter-technology handoff. The MM-PNEMO scheme is appraised via NS-3 network simulator. The handoff latency, packet loss, packet delivery ratio, and throughput have been selected as the performance metrics. In summary, the simulation outcomes recommend that car-based NEMO network set up in the proposed multihoming-based mobility management architecture presented better performance. The proposed scheme attained an improved handoff performance as illustrated in Table 4. In the course of the simulation, the proposed scheme attained a lower number of packet loss and handoff delay. This outcome is potential since the proposed MM-PNEMO scheme is capable of splitting the application flow burden among multiple access technology. Hence, it can be concluded from evaluation outcomes that, the performance of multihoming-based scheme (MM-PNEMO) specifies better compared to single-homed scheme (NEMO BSP and PNEMO).

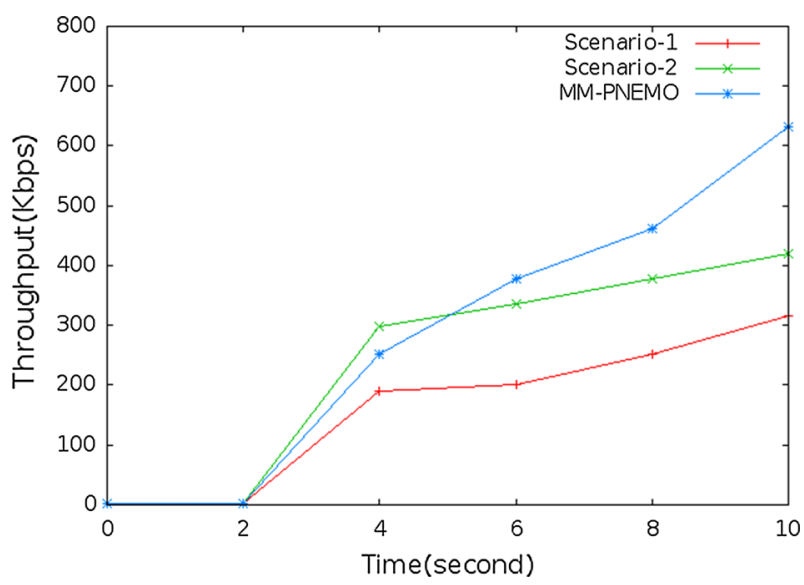

Fig. 13 Throughput versus time 
Table 4 Summary of results analysis in simulation approach

\begin{tabular}{llll}
\hline Performance metrics & $\begin{array}{l}\text { Scenario 1 } \\
\text { (NEMO BSP) }\end{array}$ & $\begin{array}{l}\text { Scenario 2 } \\
\text { (PNEMO) }\end{array}$ & $\begin{array}{l}\text { Scenario 3 } \\
\text { (MM-PNEMO) }\end{array}$ \\
\hline Handoff delay (s) & 1.2 & .51 & .20 \\
Average packet loss & 31 & 26 & 4 \\
Packet loss ratio (\%) & 13 & 5 & 1 \\
Packet delivery ratio (\%) & 87 & 95 & 99 \\
Throughput (kbps) & 316 & 421 & 631 \\
\hline
\end{tabular}

\section{Conclusion}

This paper proposed an innovative multihoming-based scheme to support flow-based mobility in PNEMO environment. The functionalities of the network entity (LMA), mobility entity (MR) and signaling messages has been enhanced to support flow-based fast registration mechanism. This is to ensure seamless handoff during inter-technology handoff. Simulation approaches used to evaluate the proposed scheme. In simulation part, NS-3 has been used where handoff latency, packet loss, packet delivery ratio, and throughput have been considered as performance metrics. The proposed scheme has been compared with the standard NEMO BSP and PNEMO for benchmarking. The obtained results show that, the proposed scheme has achieved significant improvement by maximizing the packet delivery ratio (around 99\%) and reducing the handoff delay comparing to the standard NEMO BSP and PNEMO. The reason is that the SMR in the proposed scheme is capable of selecting specific flows via using multiple interfaces simultaneously except involving buffering mechanism. As a result, it is not essential to contest with other application flows. The network is retained more load balanced. The experimental testbed is required to include as a future recommendation in order to precise assessment on the endto-end delay of the MM-PNEMO scheme.

Acknowledgements A special thanks to the Government of Malaysia, through Ministry of Higher Education (MoHE) for the education sponsorship, and I equally appreciate International Islamic University Malaysia through Research Management Centre (RMC).

\section{References}

1. Hossain, M. S., Atiquzzaman, M. \& Ivancic, W. (2012). Performance evaluation of multihomed NEMO. In Communications (ICC), 2012 IEEE international conference on. IEEE.

2. Hossain, M. S., Atiquzzaman, M. \& W. Ivancic. Performance comparison between multihomed network mobility protocols. In Global communications conference (GLOBECOM), 2012 IEEE. IEEE.

3. Devarapalli, V., Wakikawa, R., Petrescu, A., \& Thubert, P. (2005). Network mobility (NEMO) basic support protocol, IETF RFC 3963.

4. Hossain, M. S., Atiquzzaman, M., \& Ivancic, W. (2011). Cost analysis of mobility management entities of SINEMO. In Communications (ICC), 2011 IEEE international conference on. IEEE.

5. Lee, J.-H., \& Ernst, T. (2011). Lightweight network mobility within PMIPv6 for transportation systems. Systems Journal, IEEE, 5(3), 352-361.

6. Lee, J.-H., Ernst, T., \& Chilamkurti, N. (2012). Performance analysis of PMIPv6-based network mobility for intelligent transportation systems. Vehicular Technology, IEEE Transactions on, 61(1), 74-85. 
7. Lee, C.-W., Chen, M. C., \& Sun, Y. S. (2014). A novel network mobility management scheme supporting seamless handover for high-speed trains. Computer Communications, 37, 53-63.

8. Wakikawa, R., Devarapalli, V., Tsirtsis, G., Ernst, T., \& Nagami, K. (2009). Multiple care-of addresses registration. IETF RFC 5648.

9. Tsirtsis, G., Soliman, H., Montavont, N., Giaretta, G., \& Kuladinithi, K. (2011). Flow bindings in mobile IPv6 and network mobility basic support protocol (NEMO BSP). IETF RFC 6089.-95.

10. Meneguette, R. I., Bittencourt, L. F., \& Madeira, E. R. M. (2013). A seamless flow mobility management architecture for vehicular communication networks. Communications and Networks, Journal of, 15(2), 207-216.

11. Calderon, M., Bernardos, C., \& Soto, I. (2012). PMIPv6 and network mobility problem statement. IETF. draft-bernardos-netext-pmipv6-nemo-ps-02.

12. Krishnan, S., et al. (2009). Issues with network based inter-technology handovers. IETF draft-krishnannetextintertech-ps-02.

13. Ryu, S., Park, K. J., \& Choi, J. W. (2014). Enhanced fast handover for network mobility in intelligent transportation systems. Vehicular Technology, IEEE Transactions on, 63(1), 357-371.

14. Choi, J.-I., Seo, W.-K., \& Cho, Y.-Z. (2015). Efficient network mobility support scheme for proxy mobile IPv6. EURASIP Journal on Wireless Communications and Networking, 2015(1), 1-13.

15. Kuntz, R., Montavont, J., \& Noel, T. (2013). Multihoming in IPv6 mobile networks: progress, challenges, and solutions. Communications Magazine, IEEE, 51(1), 128-135.

16. Park, H.-D., \& Park, K.-N. (2013). A multihoming-based vertical handover scheme. In K. J. Kim \& K.Y. Chung (Eds.), IT Convergence and Security 2012 (pp. 749-754). Dordrecht: Springer.

17. Chen, X., et al. (2010). Experimentation and performance analysis of multi-interfaced mobile router scheme. Simulation Modelling Practice and Theory, 18(4), 407-415.

18. Bernardos, C. (2016). Proxy mobile IPv6 extensions to support flow mobility. IETF. draft-ietf-netextpmipv6-flowmob-16

19. Choi, H.-Y., et al. (2012). Design and simulation of a flow mobility scheme based on proxy mobile IPv6. JIPS, 8(4), 603-620.

20. Kim, J., Morioka, Y., \& Hagiwara, J. (2012). An optimized seamless IP flow mobility management architecture for traffic offloading. In: Network operations and management symposium (NOMS), 2012 IEEE. IEEE.

21. Melia, T., et al. (2011). IP flow mobility in PMIPv6 based networks: solution design and experimental evaluation. Wireless Personal Communications, 61(4), 603-627.

22. Choi, H.-Y., et al. (2010). Implementation and evaluation of proxy mobile IPv6 in NS-3 network simulator. In Ubiquitous information technologies and applications (CUTE), 2010 proceedings of the 5th international conference on. IEEE.

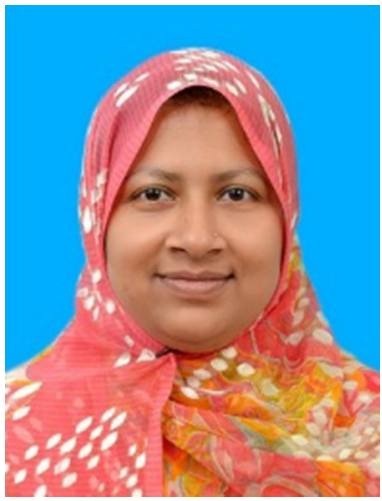

Shayla Islam received Ph.D. degree in Electrical and Computer Engineering from International Islamic University, Malaysia (IIUM) in 2016 under Malaysian International Scholarship (MIS). She completed her M.Sc. at the department of Electrical and Computer Engineering in International Islamic University, Malaysia in 2012. She completed her BSc. in Computer Science and Engineering from International Islamic University Chittagong, Bangladesh. She is currently appointed as postdoctoral research fellow at Faculty of Engineering, International Islamic University, Malaysia. Previously, she was appointed as a lecturer in Green University of Bangladesh (GUB) from 2007 to 2009. Her current research interests include network mobility, computer networks and wireless communication. She published more than 50 papers in international journals and conferences. She works as a reviewer for many international conferences as well as journals. 

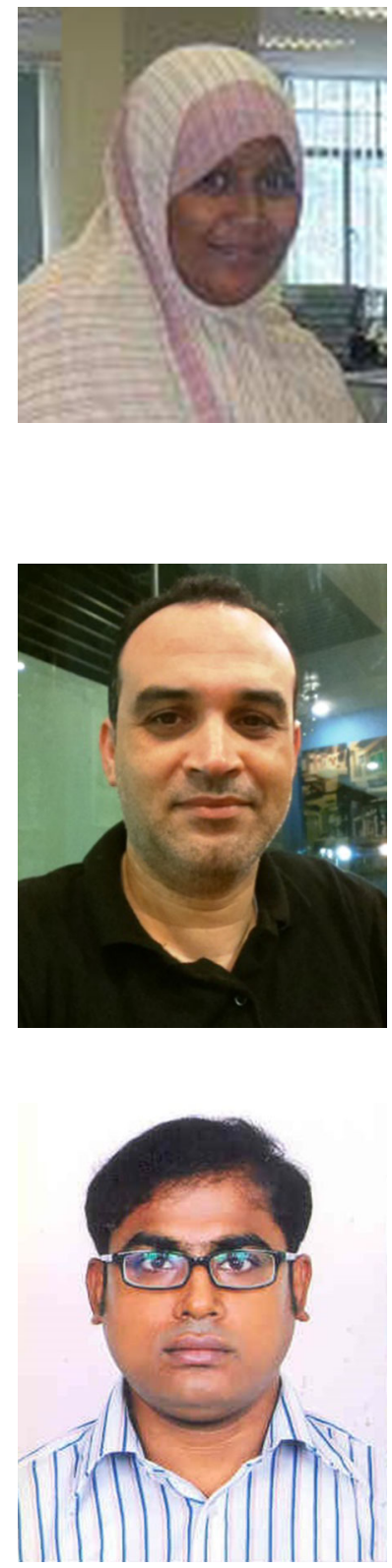

Aisha-Hassan Abdalla Hashim is currently a Professor at the Department of Electrical and Computer Engineering, Faculty of Engineering, International Islamic University Malaysia. She completed his bachelor degree studies in Electrical Engineering at University of Gezira, Sudan. She completed her M.Sc and Ph.D in Electrical and Computer Engineering from International Islamic University Malaysia. Her research interests include Wireless Networking, Network Mobility, and Cognitive Radio. She is a registered senior member of the IEEE.

Mohamed Hadi Habaebi received his B.S. degree from the Civil Aviation and Meteorology High Institute (CAHI), Libya in 1991, his M.Sc. degree in electrical engineering from Universiti Teknologi Malaysia in 1994, and his Ph.D. degree in computer and communication system engineering from University Putra Malaysia in 2001. He is currently an associate professor and post graduate academic advisor at the Department of Electrical and Computer Engineering, International Islamic University Malaysia. He heads the research works on Internet of things at the Department. He has supervised many students to obtain their Ph.D. and M.Sc. degrees, published more than 120 articles and papers, and sits on the editorial board of many international journals. His research interests include M2M communication protocols, wireless sensor and actuator networks, cognitive radio, small antenna system and radio propagation, and wireless communications and network performance evaluation. He is an active member of IEEE and an active reviewer with many international journals.

Mohammad Kamrul Hasan is currently appointed as a Post-Doctoral Research Fellow at the department of Electrical and Computer Engineering in International Islamic University, Malaysia. He has completed his Ph.D. in 2016, at the department of Electrical and Computer Engineering, International Islamic University, Malaysia. He achieved his Masters in Communication Engineering from International Islamic University, Malaysia in 2012. He has completed his B.Sc in Computer Science and Engineering in 2003, Bangladesh. His current research interests include OFDMA, Interference, Cognitive Network, Optimization, Big Data, Smart Grid Computing, and Data Communication and Networking. He published more than 50 papers in international journals and conferences. He is a Member of Institute of Electrical and Electronics Engineers (MIEEE), and Member of Institution of Engineering and Technology (MIET). 